Copyright (C) 2008 IEEE. Reprinted from

IEEE International Parallel and Distributed Processing Symposium

(21st : 2007 : Long Beach, CA, USA)

This material is posted here with permission of the IEEE. Such permission of the IEEE does not in any way imply IEEE endorsement of any of the University of Adelaide's products or services. Internal or personal use of this material is permitted. However, permission to reprint/republish this material for advertising or promotional purposes or for creating new collective works for resale or redistribution must be obtained from the IEEE by writing to pubs-permissions@ieee.org.

By choosing to view this document, you agree to all provisions of the copyright laws protecting it. 


\title{
Optimal Energy Balanced Data Gathering in Wireless Sensor Networks
}

\author{
Haibo Zhang ${ }^{1,2}$, Hong Shen ${ }^{1}$, and Yasuo Tan ${ }^{2}$ \\ ${ }^{1}$ University of Adelaide \\ ${ }^{2}$ Japan Advanced Institute of Science and Technology \\ Graduate School of Information Science \\ School of Computer Science \\ 1-1, Asahidai, Nomi, Ishikawa 923-1292, Japan \\ Adelaide, SA 5005 Australia \\ haibozh@gmail.com \\ hong@cs.adelaide.edu.au \\ ytan@jaist.ac.jp
}

\begin{abstract}
Unbalanced energy consumption is an inherent problem in wireless sensor networks where some nodes may be overused and die out early, resulting in a short network lifetime. In this paper, we investigate the problem of balancing energy consumption for data gathering sensor networks. Our key idea is to exploit the tradeoff between hop-by-hop transmission and direct transmission to balance energy dissipation among sensor nodes. By assigning each node a transmission probability which controls the ratio between hop-by-hop transmission and direct transmission, we formulate the energy consumption balancing problem as an optimal transmission probability allocation problem. We discuss this problem for both chain networks and general networks. Moreover, we present the solution to compute the optimal number of sections in terms of maximizing the network lifetime. Numerical results demonstrate that our methods outperform the traditional hop-by-hop and direct transmission schemes and achieve significant lifetime extension especially for dense sensor networks.
\end{abstract}

\section{Introduction}

Rapid advances in wireless technologies have enabled the deployment of wireless sensor networks (WSNs) for a wide range of applications such as environment monitoring (e.g., habitat, traffic and security)[3][11], industrial sensing[10] and health data gathering[1]. Sensors in such networks are usually equipped with small inexpensive batteries and expected to operate for months or even years. Moreover, in many scenarios, it is usually impractical or infeasible to replace or recharge the batteries once the sensors have been deployed. Thus, a critical consideration in de-

$\overline{1-4244-0910-1 / 07 / \$ 20.00 ~(C) 2007 ~ I E E E . ~}$ signing such WSNs is conserving energy so as to maximize the post-deployment network lifetime.

The research for energy-efficient solutions has led to numerous algorithms and protocols that strike for the goal of reducing energy consumption and prolonging network lifetime. Topology control[4][13] aims to conserve energy by dynamically adjusting the transmission power level while maintaining network connectivity. Power-aware routing protocols[12][17] reduce energy consumption by selecting minimum-energy paths to deliver packets. While sleep management schemes[19][5] save energy by periodically turning off the radio to avoid idle listening. However, all these protocols and algorithms do not explicitly take care of the phenomena of unbalanced energy consumption, which is an inherent problem in WSNs. For example, in periodical data gathering applications, multi-hop wireless paths are usually utilized to transport packets to the sink. Inevitably, sensors close to the sink may experience more traffic and higher energy consumption rate. These nodes will die out early, thus resulting in network collapse although there may be still significant amount of energy in other sensors.

Direct transmission and hop-by-hop transmission are two basic communication patterns in wireless networks. In direct transmission where packets are directly transmitted to the sink without any relay, the nodes located farther away from the sink have higher energy burden due to long range communication, and these nodes may die out first. On the other hand, the hop-by-hop transmission drains less power at each hop, but the sensors close to the sink have a high load of packets relay and may run out of energy quickly. To achieve balanced energy consumption, an elegant solution is to deliver packets through the combination of direct transmission and hop-by-hop transmission. In this article, we address the problems of balancing energy consumption and maximizing network lifetime by exploiting the energy tradeoff between direct transmission and hop-by-hop transmission. Our analysis pertains only to the data gathering 
sensor networks where each sensor must periodically report its observations to the sink.

We first discuss the problem of balancing energy consumption on chain networks and propose a fully distributed solution which exchanges only $O(1)$ messages per channel and $O(n)$ messages in total. We derive the energy balanced solution for general topology networks by dividing the network into sections and approximately mapping it on chain models. We also present the approach to compute the optimal number of sections in terms of maximizing network lifetime. Finally, we discuss the application of our scheme to heterogeneous cluster-based senor networks and show that our scheme outperforms cluster heads rotation scheme since the overheads incurred by cluster heads reelection have been completely avoided.

The rest of this article is organized as follows. Section 2 discusses related work. Section 3 describes the system models and formulates the problems. Section 4 presents the optimal energy balanced solution for data gathering in chain networks. Section 5 presents the solution for generaltopology sensor networks. Section 6 gives the approach to derive the optimal section numbers in terms of maximizing network lifetime. Section 7 discuses the application of our scheme to heterogeneous cluster sensor networks. Finally, we summarize our work and conclude the article in Section 8 .

\section{Related Work}

There have been many studies on exploring energy efficiency in wireless sensor networks. Strategies such as topology control[4][13], energy aware routing [12][17] or periodical sleeping[19][5] have been proposed to conserve energy to prolong network lifetime. However, most of them focus on minimizing the total energy consumption of endto-end packet delivery and do not explicitly solve the unbalanced energy consumption problem which can result in short network lifetime.

Clustering is commonly considered as one of the most promising techniques for prolonging network lifetime. Cluster-head rotation schemes have been proposed to balance energy consumption among the nodes within the cluster. LEACH [7] utilizes randomized rotation of clusterheads to evenly distribute the energy load. Simulations show that LEACH can achieve as much as a factor of 8 reduction in energy dissipation compared with conventional routing protocols. However, to achieve a better balance of energy consumption, the cluster-head selection algorithm must be performed frequently, which will add excessive processing and communication overheads to the network.

Communication topology control is another approach for dealing with the energy consumption balancing problem. Energy Balanced Chain (EBC) was proposed in [9] to balance energy consumption by optimizing hop distances. The communication topology is pre-determined based on the anticipated traffic within the network. Olariu and Stojmenovic[14] investigated the uneven energy depletion phenomenon in uniformly distributed sensor networks. They divide the network area into coronas and derive an iterative process to determine the optimal size of the coronas and the optimal number of coronas for balancing per-sensor energy consumption.

Power control and load balancing techniques have also been exploited to balance energy consumption in sensor networks. Perillo et al. in [15] formulated the problem of balancing energy consumption as transmission range distribution optimization problem and solved it by pre-allocating the fraction of packets for each link based on the traffic in the network. In [16], several energy-efficient communication protocols have been proposed based on power control and load balancing, aiming at even distribution of the residual energy of the sensors and thus prolonging network lifetime.

Efthymiou et al.[6] studied the problem of energybalanced data propagation in wireless sensor networks. They considered the scenario where the sensor nodes are uniformly deployed in a fan-shaped or circular region and all nodes are assumed to have the same packet generation rate. Energy balance is achieved by optimally controlling the fraction of packets for direct transmission and hop-byhop transmission. However, the authors did not actually prove that such solution always exists. In this paper, we use the same communication model and analyze the energy balancing problem for data gathering sensor networks. We also discuss the problem of computing the optimal number of sections in terms of maximizing network lifetime, and extend the solution to cluster sensor networks.

\section{System Models and Problem Statement}

We consider a sensor network composed of multiple sensor nodes and one sink node. Both the sink and sensors remain static once deployed. The sink is assumed to have continuous and sufficient power supply, while the sensors are powered by batteries that are inconvenient to replace or recharge. For each sensor, we assume that it can compute the approximate transmission distance based on the received signal strength.

\subsection{Data Gathering Model}

For most data gathering applications, the sensors usually operate in a low-duty-cycle mode. The interval between one duty cycle to the next may be several minutes, hours even days. This characteristic motivates the utilization of periodical sleeping to conserve energy. In our model, we 
assume that a loosely synchronized sleep/wakeup scheme as in S-MAC[19] and T-MAC[5] is exploited. Between two adjacent duty cycles, all sensors will turn off the radio to conserve energy.

We define the process in which all sensors wake up, generate the sensed data and send the data to the sink as one data gathering cycle (DGC). For simplicity, we do not consider data aggregation and assume that all the packets are of the same size.

\subsection{Energy Model}

It has been observed that variable-power packet transmission is more energy efficient than fixed transmission power schemes. In our model, we assume that each sensor can intelligently choose the transmission power based on the link distance. This is true in typical sensor node implementations. For example, the Berkeley Mica Mote provides such program interfaces. The first-order radio model proposed in [7] is used to describe the energy consumption for the sensors. Let $\epsilon_{t(R)}$ and $\epsilon_{r(R)}$ be the energy consumed for transmitting and receiving an $m$ bits packet over distance $R$ respectively, then

$$
\begin{gathered}
\epsilon_{t(R)}=\epsilon_{\text {elec }} \times m+\epsilon_{\text {amp }} \times m \times R^{k} \\
\epsilon_{r(R)}=\epsilon_{\text {elec }} \times m
\end{gathered}
$$

where $\epsilon_{\text {elec }}$ is the energy spent by the electronic circuit when transmitting or receiving one bit data and $\epsilon_{a m p}$ is the transmission amplifier, while $k$ is the propagation loss exponent. When receiving a packet, only the receiving circuit is invoked, therefore, the energy spent on receiving is $\epsilon_{\text {elec }} /$ bit.

\subsection{Communication Model}

The communication model we adopt is same to the one proposed in[6]. The combination of direct transmission and multi-hop transmission is exploited to balance energy consumption among the nodes. For each sensor $i$, it forwards the packets it generates or receives to next hop node towards the sink with probability $p_{i}$ and directly transmits the packets to the sink with probability $1-p_{i}$. We call $p_{i}$ the transmission probability for node $i$. Here, we simply assume that the transmission in the network is reliable and we will consider the effects of data loss in future work.

\subsection{Problem Statement}

Let $n$ represent the number of sensors in the network and $E\left[\xi_{i}\right]$ denote the expected energy consumed by node $i$ during the whole lifetime. Assume that all nodes in the network have the same amount of initial battery energy. We say that the network is energy balanced if each sensor in the network has the same expected energy consumption, i.e., when

$$
E\left[\xi_{i}\right]=E\left[\xi_{j}\right] \quad i, j=1,2, \ldots, n
$$

To achieve balanced energy consumption, each sensor should transmit its data with an optimal transmission probability. If the probability is large, transmissions tend to happen locally. The sensors close to the sink may be overused and die out early. On the other hand, if the probability is small, the large part of packets will be directly transmitted to the sink. The energy at the sensors that are far away from the sink may be exhausted quickly. Thus, the problem of balancing energy consumption can be transformed as the optimal transmission probability allocation problem. Our objective is to compute the optimal transmission probability for each node so that balanced energy consumption is achieved.

\section{Balancing Energy Consumption in Chain Networks}

We start by investigating the problem of energy balanced data gathering in chain networks. Although our final aim is to deal with this problem for general-topology networks, as we will show shortly, the analysis on chain networks provides deep insights on the solution for general networks. In this section, we discuss this problem on two different chain models.

- Regular Line Networks (RLN): where the sensors are regularly deployed along a straight line with the same distance between any two neighboring sensors.

- General Chain Networks (GCN): where the sensors are irregularly deployed in a region, and the chain is constructed by each node selecting its near-sink neighbor for next hop packet relay.

Without loss of generality, the sink is assumed to be placed at one end of the chain. The nodes are marked with 0 to $n$ from the sink to the farthermost sensor. During each data gathering cycle, node $i$ forwards its packets to node $i-1$ with probability $p_{i}$ and directly transmits the packets to the sink with probability $1-p_{i}$. The objective is to compute the optimal $p_{i}$ for node $i$ so that energy consumption is balanced among the nodes.

Consider one data gathering cycle in which each sensor wakes up, generates one packet and reports it to the sink. Let $f_{i}$ denote the number of packets that sensor $i$ forwards to sensor $i-1$ and $d_{i}$ represent the number of packets that sensor $i$ directly transmits to the sink. Thus,

$$
f_{i}+1=f_{i-1}+d_{i-1}, \quad 1<i \leq n
$$


then

Denoted by $E[\lambda]$ the expectation of a random variable $\lambda$,

$$
\text { Lemma } 1 p_{i}=\frac{E\left[f_{i}\right]}{E\left[f_{i}\right]+E\left[d_{i}\right]}, \quad 1 \leq i \leq n
$$

Proof. Let $P(i, j)(i>j>0)$ denote the probability that node $j$ receives a packet from node $i$. Obviously, the packets transmitted by node $i$ can arrive at node $j$ only through hop-by-hop transmission. Thus,

$$
P(i, j)=\prod_{k=j+1}^{i} p_{k}
$$

Since $f_{i}$ is also the number of packets received by node $i-1$. Therefore,

$$
\begin{aligned}
E\left[f_{i}\right] & =\sum_{k=i}^{n} P(k, i-1) \\
& =\sum_{k=i}^{n} \prod_{j=i}^{k} p_{j} \\
& =\sum_{k=i+1}^{n} \prod_{j=i}^{k} p_{j}+p_{i} \\
& =p_{i}\left(\sum_{k=i+1}^{n} \prod_{j=i+1}^{k} p_{j}\right)+p_{i} \\
& =p_{i}\left(\sum_{k=i+1}^{n} \prod_{j=i+1}^{k} p_{j}+1\right) \\
& =p_{i}\left(E\left[f_{i+1}\right]+1\right)
\end{aligned}
$$

Because $f_{i+1}+1=f_{i}+d_{i}$, by the linearity of expectation, $E\left[f_{i+1}\right]+1=E\left[f_{i}\right]+E\left[d_{i}\right]$. Replacing $E\left[f_{i+1}\right]+1$ with $E\left[f_{i}\right]+E\left[d_{i}\right]$, we get $p_{i}=\frac{E\left[f_{i}\right]}{E\left[f_{i}\right]+E\left[d_{i}\right]}, \quad 1 \leq i \leq n$.

Let $E\left[\varepsilon_{i}\right]$ be the expected energy consumed by sensor $i$ in one $D G C$. We get the following theorem.

Theorem $1 E\left[\xi_{i}\right]=E\left[\xi_{j}\right] \forall i, j=1,2, \ldots, n$ if and only if $E\left[\varepsilon_{i}\right]=E\left[\varepsilon_{i-1}\right]$ where $i=2, \ldots, n$.

Proof. In our scheme, the optimal transmission probability for each sensor node is pre-computed and remains constant once the network starts to work. Therefore, the performance of data gathering in one $D G C$ is fully independent on those in other $D G C s$. Let $N$ be the total number of $D G C$ in the whole lifetime, then, $E\left[\xi_{i}\right]=N E\left[\varepsilon_{i}\right]$. Therefore, $E\left[\xi_{i}\right]=E\left[\xi_{j}\right] \Leftrightarrow E\left[\varepsilon_{i}\right]=E\left[\varepsilon_{j}\right]$. By the transitive property, Theorem 1 is correct.

\subsection{Optimal Solution for RLN Case}

Consider the case where $n$ sensor nodes are regularly deployed along a straight line with equal distance $R$ between any two adjacent nodes (see Fig.1). The sink is placed at one end of the network. Therefore, the distance from node $i(1 \leq i \leq n)$ to the sink is $i R$.

Before presenting the distributed algorithm for allocating the optimal transmission probability among the nodes in $R L N$, we give the following lemma.

Lemma 2 To balance energy consumption in RLN, the following equation should hold

$$
\begin{array}{r}
E\left[d_{i}\right]=\frac{\left((i-1)^{k}-1\right) \epsilon_{a m p} R^{k} E\left[d_{i-1}\right]+2 \epsilon_{e l e c}+R^{k} \epsilon_{a m p}}{2 \epsilon_{e l e c}+i^{k} R^{k} \epsilon_{a m p}} \\
i=2, \ldots, n
\end{array}
$$

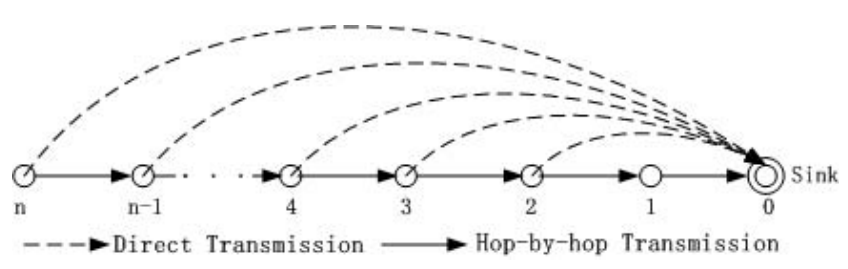

Figure 1. A regular line network composed of $n$ sensor nodes

Proof. For node $i$ where $2 \leq i \leq n$, the expected energy consumed during one $D G C$ is given by

$$
E\left[\varepsilon_{i}\right]=\underbrace{E\left[f_{i}\right] \epsilon_{t(R)}+E\left[d_{i}\right] \epsilon_{t(i R)}}_{\text {transmit energy }}+\underbrace{E\left[f_{i+1}\right] \epsilon_{r(R)}}_{\text {receive energy }}
$$

where $E\left[f_{i}\right] \epsilon_{t(R)}$ is the expected energy spent by node $i$ for forwarding packets to node $i-1$ and $E\left[d_{i}\right] \epsilon_{t(i R)}$ is the expected energy spent on transmitting the packets directly to the sink, while $E\left[f_{i+1}\right] \epsilon_{r(R)}$ represents the expected energy consumed for receiving packets from node $i+1$. Obviously, $E\left[f_{n+1}\right]=0$ since node $n$ receives no packets during data gathering.

Consider two neighboring nodes $i$ and $i-1$, from Theorem 1 we get

$$
\begin{gathered}
E\left[\varepsilon_{i}\right]=E\left[\varepsilon_{i-1}\right] \Leftrightarrow \\
\left(E\left[f_{i}\right]-E\left[f_{i-1}\right]\right) \epsilon_{t(R)}+\left(E\left[f_{i+1}\right]-E\left[f_{i}\right]\right) \epsilon_{r(R)} \\
=E\left[d_{i-1}\right] \epsilon_{t((i-1) R)}-E\left[d_{i}\right] \epsilon_{t(i R)}
\end{gathered}
$$

Note that $f_{i+1}+1=f_{i}+d_{i}$, by the linearity of expectation, $E\left[f_{i+1}\right]-E\left[f_{i}\right]=E\left[d_{i}\right]-1$. Replacing $E\left[f_{i+1}\right]-E\left[f_{i}\right]$ and $E\left[f_{i}\right]-E\left[f_{i-1}\right]$ with $E\left[d_{i}\right]-1$ and $E\left[d_{i-1}\right]-1$ respectively in Equation (10), we get

$$
E\left[d_{i}\right]=\frac{\left(\epsilon_{t(i R)}-\epsilon_{t(R)}\right) E\left[d_{i-1}\right]+\epsilon_{r(R)}+\epsilon_{t(R)}}{\epsilon_{r(R)}+\epsilon_{t(i R)}}
$$

By Equation (1) and (2),

$$
\begin{array}{r}
E\left[d_{i}\right]=\frac{\left((i-1)^{k}-1\right) \epsilon_{a m p} R^{k} E\left[d_{i-1}\right]+2 \epsilon_{\text {elec }}+R^{k} \epsilon_{a m p}}{2 \epsilon_{\text {elec }}+i^{k} R^{k} \epsilon_{a m p}} \\
i=2, \ldots, n
\end{array}
$$

For node 1 which is only one hop away from the sink, the delivery of any packet from node 1 to the sink can be regarded as either direct transmission or hop-by-hop transmission. From Lemma 2, $E\left[d_{2}\right]=\frac{2 \epsilon_{\text {elec }}+R^{k} \epsilon_{a m p}}{2 \epsilon_{\text {elec }}+2^{k} R^{k} \epsilon_{a m p}}$, which implies that $p_{1}$ will not influence the allocation of transmission probabilities for other nodes. Therefore, $p_{1}$ can be assigned any value ranging from 0 to 1 .

Note that $\epsilon_{\text {elec }}$ and $\epsilon_{a m p}$ are both system-dependent parameters. Given fixed $R, E\left[d_{i}\right](i>2)$ depends only on $E\left[d_{i-1}\right]$. Observe that $E\left[f_{i}\right]=E\left[f_{i+1}\right]+1-E\left[d_{i}\right]$, both 
$E\left[d_{i}\right]$ and $E\left[f_{i}\right]$ can be obtained by local information exchange. Therefore, the optimal transmission probability that achieves balanced energy consumption can be easily computed according to Lemma 1 . Now we present the distributed algorithm run at each node $u$ to compute optimal $p_{u}$.

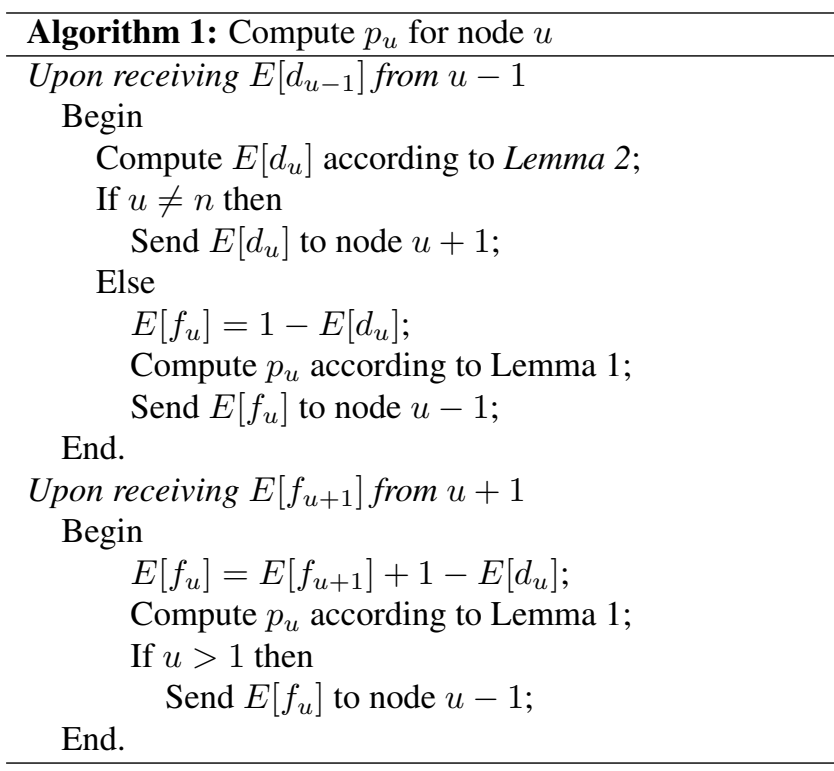

It is easy to prove that Algorithm 1 exchanges only $O(1)$ messages per-channel and $O(n)$ messages in total, which indicates its energy efficiency and suitability for resourcelimited sensor networks.

Now we give the proof that such optimal solution always exists for $R L N$.

Lemma $30<E\left[d_{i}\right]<1, \quad i=2,3, \ldots, n$.

Proof. From Lemma 2, $E\left[d_{2}\right]=\frac{2 \epsilon_{\text {elec }}+R^{k} \epsilon_{a m p}}{2 \epsilon_{\text {elec }}+2^{k} R^{k} \epsilon_{a m p}}$. Since $\epsilon_{\text {elec }}>0, \epsilon_{\text {amp }}>0$ and $k>0$, therefore, $0<E\left[d_{2}\right]<1$. Suppose $0<E\left[d_{i}\right]<1$ where $2<i<n$. Then, $E\left[d_{i+1}\right]>\frac{2 \epsilon_{\text {elec }}+R^{k} \epsilon_{a m p}}{2 \epsilon_{\text {elec }}+(i+1)^{k} R^{k} \epsilon_{a m p}}>0$, and $E\left[d_{i+1}\right]<$ $\frac{\left(i^{k}-1\right) \epsilon_{a m p} R^{k} * 1+2 \epsilon_{\text {elec }}+R^{k} \epsilon_{a m p}}{2 \epsilon_{\text {elec }}+(i+1)^{k} R^{k} \epsilon_{a m p}}<1$. Hence, the lemma is proved.

Theorem 2 There always exists an optimal transmission probability for each node in $R L N$ so that energy consumption can be balanced during data gathering.

Proof. If there exists a solution that can achieve balanced energy consumption for data gathering in $R L N$, the following conditions must be satisfied.

1. $E\left[\xi_{i}\right]=E\left[\xi_{j}\right], \quad 1 \leq i, j \leq n$.

2. $0 \leq p_{i} \leq 1, \quad 1 \leq i \leq n$.
From the proof of Lemma 2 and Theorem 1, $p_{i}$ obtained by Algorithm 1 must satisfy the first condition. From Lemma $3,0<E\left[d_{n}\right]<1$, and $E\left[f_{n}\right]=1-E\left[d_{n}\right]>0$. Suppose $E\left[f_{i}\right]>0$ where $1<i<n$. Since $E\left[f_{i}\right]=E\left[f_{i-1}\right]+$ $E\left[d_{i-1}\right]-1$ and $0<E\left[d_{i-1}\right]<1$, we can get $E\left[f_{i-1}\right] \geq 0$. From Lemma 3 and Lemma $1,0 \leq p_{i} \leq 1$. Hence, the theorem is proved.

\subsection{Solution for GCN Case}

Despite the merits of $R L N$ model such as simplicity and existence of optimal solution for energy balanced consumption, it has too much constraints on network deployment. In practice, it may be not suitable to deploy the sensors along a straight line with equal distance. In this subsection, we focus on solving the balancing energy consumption problem for more general chain network.

Consider a simple example of GCN (Fig. 2). Let $R_{i}$ represent the distance from node $i$ to the sink. To derive the energy balancing solution, we also assume that all the sensors use the same power for hop-by-hop packet transmission. The assumption is reasonable in view of the following two practical considerations. First, for homogeneous sensor networks, two neighboring sensors may not be placed too close or too far in view of network coverage. More importantly, most current sensor motes can not transit a packet with power as small as possible. Usually, there is a minimum transmission power. In some scenarios, all sensors have to use this minimum power for hop-by-hop packet transmission.

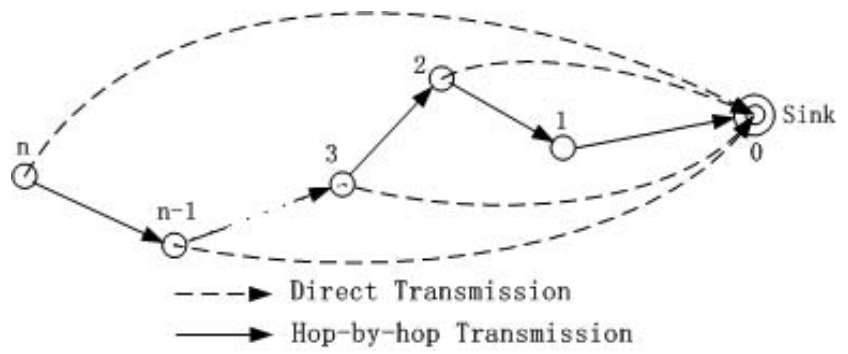

\section{Figure 2. A general chain network composed of $\mathbf{n}$ sensor nodes}

Given fixed transmission power $P$ for hop-by-hop packet forwarding, let $R_{0}$ be the maximum distance that a packet can be transmitted using $P$. Denoted by $R_{i, j}$ the distance from node $i$ to node $j$. Obviously, $R_{0} \geq \operatorname{Max}\left\{R_{i, i-1}, 1 \leq\right.$ $i \leq n\}$. Similar to $R L N$, we can get the following lemma.

Lemma 4 To balance energy consumption in $\mathrm{GCN}$, the following equation should hold:

$$
E\left[d_{i}\right]=\frac{\left(R_{i-1}^{k}-R_{0}^{k}\right) \epsilon_{a m p} E\left[d_{i-1}\right]+2 \epsilon_{e l e c}+R_{0}^{k} \epsilon_{a m p}}{2 \epsilon_{\text {elec }}+R_{i}^{k} \epsilon_{a m p}}
$$




$$
i=2, \ldots, n
$$

The proof of Lemma 4 is similar to that of Lemma 2 and we omit it here.

If $R_{i} \geq R_{i-1} \geq R_{0}(1<i \leq n)$ which can be guaranteed during the chain construction, the optimal solution for balancing energy consumption in $G C N$ always exists. Even when $R_{1}<R_{0}$, the optimal solution may still exist if $R_{1}$ is not too small. We will demonstrate this by numerical results later. Obviously, the optimal transmission probability for each node in $G C N$ can also be computed by Algorithm 1.

\subsection{Numerical Results and Analysis}

We set $k=2, n=100, \epsilon_{\text {elec }}=50 \mathrm{~nJ} /$ bit and $\epsilon_{\text {amp }}=$ $100 \mathrm{pJ} / \mathrm{bit} / \mathrm{m}^{2}$. Fig. 3 plots the optimal transmission probability obtained by Algorithm 1 for each node in a $R L N$. For all cases, it can be observed that the optimal transmission probability increases initially, and then remains more or less for a large number of nodes in the middle part of the network. Finally, it drops quickly for the nodes located at the end part of the network. This behavior can be explained based on the energy model we adopt. For the nodes that are far away from the sink, it handles only a small number of packets. To balanced energy consumption, direct transmission will be used frequently to deliver the packets. While for the node that are close to the sink, although the distance to the sink is small, the probability still remains large because the nodes need to relay more packets compared with the nodes at the middle and last part.

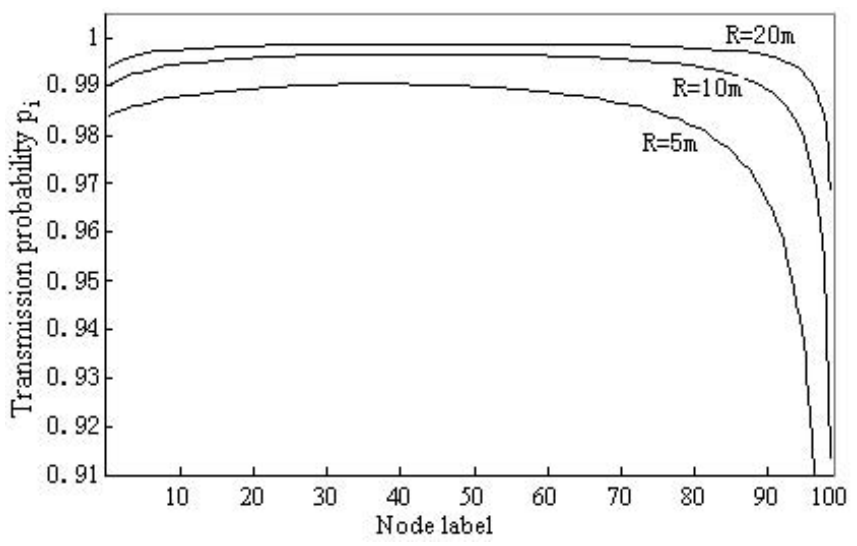

Figure 3. The transmission probability $p_{i}$ for sensor $i$ in $R L N$ with $k=2, n=100, \epsilon_{\text {elec }}=$ $50 \mathrm{~nJ} / \mathrm{bit}$ and $\epsilon_{a m p}=100 \mathrm{pJ} / \mathrm{bit} / \mathrm{m}^{2}$.

Fig.4 shows the optimal transmission probability obtained for each node in a $G C N$ with 100 nodes. The system parameters, $k, \epsilon_{e l e c}$ and $\epsilon_{a m p}$, are chosen to be the same

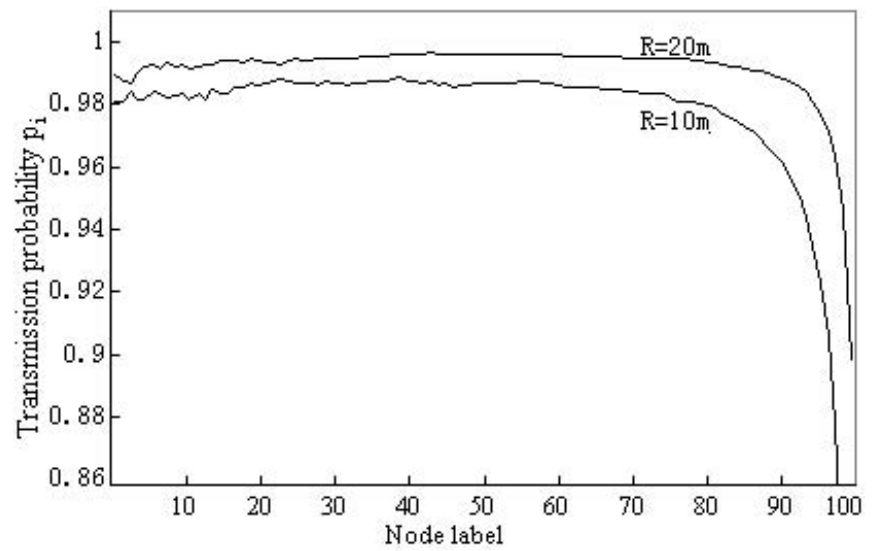

Figure 4. The transmission probability $p_{i}$ for sensor $i$ in $G C N$ with $k=2, n=100, \epsilon_{\text {elec }}=$ $50 \mathrm{~nJ} / \mathrm{bit}$ and $\epsilon_{a m p}=100 \mathrm{pJ} / \mathrm{bit} / \mathrm{m}^{2}$.

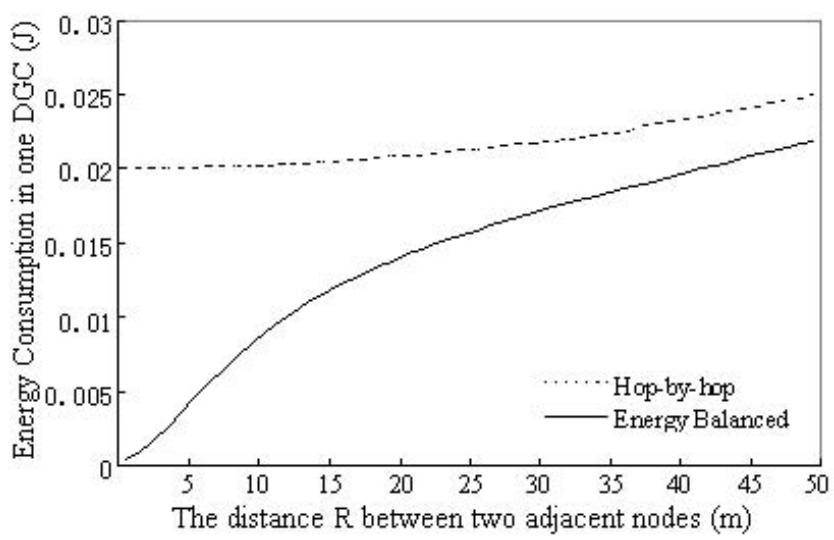

$$
\begin{aligned}
& \text { Figure 5. Comparison of energy consumption } \\
& \text { for node } 1 \text { in } R L N \text { with } n=100, \epsilon_{\text {elec }}=50 \mathrm{~nJ} / \mathrm{bit} \\
& \text { and } \epsilon_{a m p}=100 \mathrm{pJ} / \mathrm{bit} / \mathrm{m}^{2} \text {. }
\end{aligned}
$$

as those in $R L N$. The distance from node $i$ to the sink is initiated using the following function which guarantees that $R_{i} \geq R_{0},(i \geq 1) . R_{i+1}=R_{i}+\operatorname{Random}\left(R_{0}\right), R_{0}>0$. As seen from Fig. 4 , the transmission probability has the same change trend as that in $R L N$ although the curve is not smooth. It is also deserve to note that the transmission probabilities for the nodes close to the sink remain large (e.g., larger than 0.9). Therefore, even when $R_{1}<R_{0}$, the optimal solution may still exist.

Delivering packets through multi-hop routes with short hop-to-hop distance has been shown more energy efficient than single hop with long distance. To demonstrate the efficiency of our scheme, we compare it with hop-by-hop transmission scheme on a $R L N$ composed of 100 nodes. Fig. 5 shows the energy consumed by node 1 in one $D G C$ 
with respect to the variation of hop distance R. Clearly, our energy balanced solution consumes less energy than hopby-hop transmission scheme especially when the distance $R$ is relatively small. Nearly $82 \%$ of energy saving has been achieved by our scheme when $R=5 \mathrm{~m}$. Even when $R=20 \mathrm{~m}$, our scheme can still save more than $34 \%$ of energy than the hop-by-hop transmission scheme. However, with the increase of $R$, the improvement degrades since the direct transmission for most nodes become energy inefficient and most packets will be delivered via hop-by-hop transmission.

\section{Balancing Energy Consumption in General Topology Sensor Networks}

In general, a large number of sensors may be deployed in a region to perform data gathering task. The sensors selforganize into a network and the topology may be more complex than a simple chain. In this section, we discuss energy balanced data gathering for networks deployed in a striplike region with general topology. However, this constraint may be removed by combing our scheme with clustering techniques and we will discuss this improvement in Section 7.

Consider a snapshot of the general topology sensor network with $n$ sensor nodes and one sink. We divide the network into $c$ sections where each section contains the same number of sensors, i.e., $\frac{n}{c}$. For simplicity, we assume that $n$ can always be divided by $c$. A simple example is given in Fig. 6.

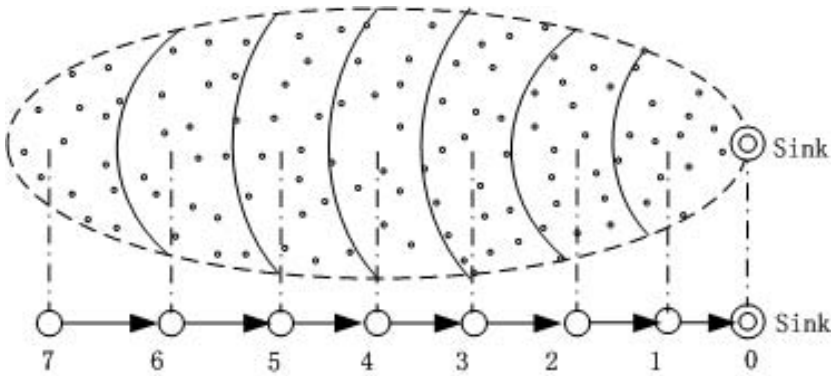

Figure 6. A general wireless sensor network and its map onto the chain model

Let $S_{i}$ denote the $i t h$ section and $R_{S_{i}}$ represent the distance from $S_{i}$ to the sink. $R_{S_{i}}$ is defined as the maximum distance from sensors in $S_{i}$ to the sink. To derive the energy balanced solution, we make the following assumptions:

1. All sensors in $S_{i}$ forward their packets to sensors in $S_{i-1}$ with same probability $p_{i}$ and transmit packets directly to the sink with same probability $1-p_{i}$.
2. All sensors in $S_{i}$ use the same power to forward packets to sensors in $S_{i-1}$. Similarly, all sensors in $S_{i}$ use the same power to transmit packets directly to the sink.

3. For all sensors in the same section, the expected number of packets received in one $D G C$ is same.

The last assumption seems strict, but it is not difficult to realize. Since each section contains the same number of sensors, there must exist the following bijective mapping between any two adjacent sections $S_{i}$ and $S_{i-1}$.

$$
\psi: k \rightarrow \psi(k) \quad k \in S_{i}, \quad \psi(k) \in S_{i-1}
$$

When node $k$ forwards a packet to next section, the packet is unicasted to $\psi(k)$ instead of broadcasting. By this, the last assumption can be guaranteed.

Let $\operatorname{dis}(j, k)$ represent the distance from node $j$ to node $k$ where $j \in S_{i}$ and $k \in S_{i-1}$. From the second assumption, the optimal bijective map between $S_{i}$ and $S_{i-1}$ can be obtained by solving the following optimization problem.

$$
\text { Minimize } \max _{j \in S_{i}, k \in S_{i-1}} \operatorname{dis}(j, k)
$$

Based on these assumptions, we can get the following lemma.

Lemma $5 E\left[\epsilon_{j}\right]=E\left[\epsilon_{k}\right], \forall j, k \in S_{i} \quad 1 \leq i \leq c$

Proof. Let $h_{i}$ denote the number of packets handled by node $i$ in one $D G C$. From the second assumption, the energy spent by all sensors in the same section on forwarding a packet to next section is same, and we denote it by $\epsilon$. Thus, the expected energy spent by node $j \in S_{i}$ in one $D G C$ is

$$
\begin{aligned}
E\left[\varepsilon_{j}\right]=p_{i} E\left[h_{j}\right] \epsilon+ & \left(1-p_{i}\right) E\left[h_{j}\right] \epsilon_{t\left(R_{S_{i}}\right)} \\
& +\left(E\left[h_{j}\right]-1\right) \epsilon_{r\left(R_{S_{i}}\right)}
\end{aligned}
$$

where $p_{i} E\left[h_{j}\right] \epsilon$ is the expected energy spent on forwarding packets to next section and $\left(1-p_{i}\right) E\left[h_{j}\right] \epsilon_{t\left(R_{S_{i}}\right)}$ is the expected energy consumed for direct transmission, while $\left(E\left[h_{j}\right]-1\right) \epsilon_{r\left(R_{S_{i}}\right)}$ represents the energy consumed for packets receiving.

For any node $j$ in the last section $S_{c}, h_{j}=1$. From Equation (13), all nodes in $S_{c}$ have the same energy consumption.

For any $j, k \in S_{i}$ where $1 \leq i<c$, from the third assumption, $E\left[f_{j}\right]=E\left[f_{k}\right]$. Since $E\left[h_{i}\right]=E\left[f_{i}\right]+1$, we get $E\left[h_{j}\right]=E\left[h_{k}\right]$. From Equation (13), $E\left[\varepsilon_{j}\right]=E\left[\varepsilon_{k}\right]$.

By Lemma 5, the energy consumption for the nodes that fall in the same section have been balanced. If energy consumption for the nodes located in different sections can be balanced, the objective is achieved. Since all nodes in the same section have the same probability for packets forwarding or direct transmission, the network can be approximately mapped onto the chain model if we look upon each 
section as one node (also see Fig.6), where each node generates $\frac{n}{c}$ packets per $D G C$.

Consider the case where all nodes are uniformly deployed in a regular region such as square or strip. The distance between any two adjacent sections is same, denoted by $R$. Therefore, the network can be mapped onto the $R L N$ model. Let $D_{i}$ denote the number of packets that the sensors in $S_{i}$ directly transmit to the sink. Similar to Lemma 2 ,

$$
\begin{array}{r}
E\left[D_{i}\right]=\frac{\left((i-1)^{k}-1\right) \epsilon_{a m p} R^{k} E\left[D_{i-1}\right]+\frac{2 n}{c} \epsilon_{e l e c}+\frac{n}{c} R^{k} \epsilon_{a m p}}{2 \epsilon_{e l e c}+i^{k} R^{k} \epsilon_{a m p}} \quad i=2, \ldots, c
\end{array}
$$

It is easy to prove that $0<E\left[D_{i}\right]<\frac{n}{c}$. From Theorem 2, the optimal $p_{i}$ for each section $S_{i}$ always exists.

For the case where the distance between two adjacent sections is not equal. The solution can be obtained by mapping the network onto the $G C N$ model.

\section{Maximizing Network Lifetime}

In this section, we focus on deriving the optimal number of sections with the objective to maximize the network lifetime. Similar to other studies[2][8], the network lifetime is defined as the time elapsed until the first sensor node in the network runs out of its energy. We measure the network lifetime by the number of $D G C$ that the network can functionally operate.

It seems as if the network lifetime can be maximized once energy dissipation has been balanced among the nodes in the network. The case is true for fixed transmission power scenario. But for variable transmission power case, the network lifetime also depends on the transmission power which determines the network topology. In our scheme, both the hop-by-hop transmission power and the direct transmission power can be controlled by adjusting the number of sections. If the network is divided into only a few sections, the distance between two adjacent sections is large. The expected energy consumption may be high since the energy spent on transmitting a packet over distance $R$ is proportional to $R^{k}$. On the other hand, dividing the network into a large number of small sections decreases the energy dissipation for hop transmission, but increases the number of hops. As shown in the energy model, a fixed amount of energy $\left(\epsilon_{\text {elec }} J / b i t\right)$ will be spent to run the electronic circuit whenever transmitting or receiving a packet. Thus, a large amount of energy may be wasted by running the electronic circuits, resulting in the increase of the expected energy consumption. Therefore, there is a trade off for choosing the number of sections in the view point of network lifetime maximization.

As shown in Section 5, the solution for balancing energy consumption in general-topology networks can be derived by mapping it onto the chain model. Thus, the optimal section number problem can be discussed on chain networks.

Consider a chain network with $n$ sensor nodes. The network is divided into $c$ sections and each section contains $\frac{n}{c}$ nodes. Since energy consumption is balanced, the expected energy consumption for any node in one $D G C$ is equal to that of the last node, i.e., $E\left[\varepsilon_{n}\right]$. Therefore, the network lifetime can be maximized only when $E\left[\varepsilon_{n}\right]$ is minimized, and the problem can be formulated as the following optimization problem.

$$
\begin{array}{r}
\text { Minimize } \quad E\left[\varepsilon_{n}\right] \\
\text { s.t. } \quad 1 \leq c \leq n
\end{array}
$$

Let $E\left[D_{i}\right]$ represent the expected number of packets forwarded from $S_{i}$ to $S_{i-1}$. From the analysis in Section 5, for any node $j$ in $S_{i}, E\left[d_{j}\right]=\frac{c E\left[D_{i}\right]}{n}$.

For $R L N$ model, let $L$ be the distance from node $n$ to the sink. Then, the expected energy consumed by node $n$ can be computed by the following equation.

$$
E\left[\varepsilon_{n}\right]=\left(1-E\left[d_{n}\right]\right) \epsilon_{t\left(\frac{L}{c}\right)}+E\left[d_{n}\right] \epsilon_{t(L)}
$$

where $\left(1-E\left[d_{n}\right]\right) \epsilon_{t\left(\frac{L}{c}\right)}$ is the energy spent on packets forwarding, while $E\left[d_{n}\right] \epsilon_{t(L)}$ represents the energy consumed for direct transmission. Since node $n$ doesn't receive any packets during data gathering, the energy consumed for receiving packets is 0 .

Replacing $E\left[d_{n}\right]$ with $\frac{c E\left[D_{c}\right]}{n}$ in Equation (16), by Equation (1) and (2), we get

$E\left[\varepsilon_{n}\right]=m \epsilon_{a m p} L^{k}\left[\left(1-\frac{c}{n} E\left[D_{c}\right]\right)\left(\frac{1}{c^{k}}-1\right)+1\right]+m \epsilon_{\text {elec }}$

From Equation (14),

$$
E\left[D_{c}\right]=\frac{\left((c-1)^{k}-1\right)\left(\frac{L}{c}\right)^{k} E\left[D_{c-1}\right] \epsilon_{a m p}+\frac{2 n}{c} \epsilon_{\text {elec }}+\frac{n}{c}\left(\frac{L}{c}\right)^{k} \epsilon_{a m p}}{2 \epsilon_{\text {elec }}+L^{k} \epsilon_{a m p}}
$$

For $G C N$ model, we assume that all nodes use the same power for packets forwarding. Let $R_{S_{0}}$ be the maximal distance between two adjacent sections. Similarly, $E\left[\varepsilon_{n}\right]$ and $E\left[D_{c}\right]$ can be computed by the following equations.

$$
\begin{array}{r}
E\left[\varepsilon_{n}\right]=E\left[d_{n}\right] \epsilon_{t\left(R_{S_{c}}\right)}+\left(1-E\left[d_{n}\right]\right) \epsilon_{t\left(R_{S_{0}}\right)} \\
=m \epsilon_{a m p}\left[\left(1-\frac{c}{n} E\left[D_{c}\right]\right)\left(R_{S_{0}}^{k}-R_{S_{c}}^{k}\right)+R_{S_{c}}^{k}\right]+m \epsilon_{\text {elec }} \\
E\left[D_{c}\right]=\frac{\left(R_{S_{c-1}}^{k}-R_{S_{0}}^{k}\right) \epsilon_{a m p} E\left[D_{c-1}\right]+\frac{2 n}{c} \epsilon_{\text {elec }}+\frac{n}{c} R_{S_{0}}^{k} \epsilon_{a m p}}{2 \epsilon_{\text {elec }}+R_{S_{c}}^{k} \epsilon_{a m p}}
\end{array}
$$

For both cases, it is difficult to obtain a closed form expression for $E\left[\varepsilon_{n}\right]$. Hence, we solve the problem numerically by the recursive random search (RRS) algorithm proposed in [18] which overcomes the drawbacks of random search and can provide a strong probabilistic and fast convergence. 


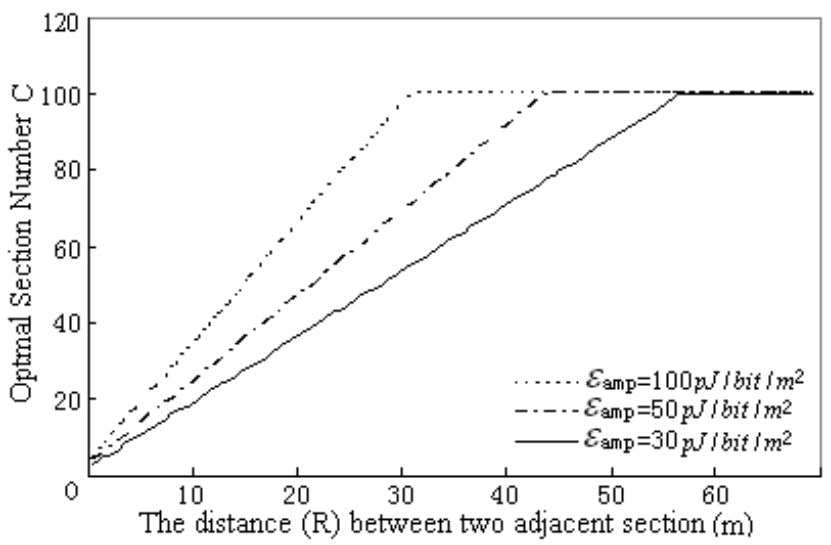

Figure 7. Optimal section number $c$ for $R L N$ with $n=100, k=2$ and $\epsilon_{\text {elec }}=50 \mathrm{~nJ} / \mathrm{bit} / \mathrm{m}^{2}$.

Fig.7 plots the optimal number of sections obtained on a $R L N$ composed of 100 nodes with different $\epsilon_{a m p}$. We set $k=2, \epsilon_{\text {elec }}=50 \mathrm{~nJ} /$ bit and the hop distance, $R$, was varied from $1 \mathrm{~m}$ to $70 \mathrm{~m}$. Interestingly, the optimal section number increases almost linearly before reaching $n$ with the increase of hop distance R. While for the same hop distance, the optimal section number obtained by a small $\epsilon_{a m p}$ is smaller than that obtained with a big $\epsilon_{a m p}$.

Let $\varpi$ be the initial energy supply for each sensor. In hop-by-hop transmission, the first node will experience the heaviest traffic burden and die out firstly. Let $\varepsilon_{1}$ be energy consumed in one $D G C$, the lifetime is $\frac{\varpi}{\varepsilon_{1}}$ for hop-by-hop transmission according to the definition of network lifetime. While for our scheme, network lifetime is $\frac{\varpi}{E\left[\varepsilon_{n}\right]}$ since energy consumption has been balanced. We set $\varpi=30 J$. Fig. 8 gives the comparison of lifetime for energy balanced scheme and hop-by-hop transmission scheme when $k=$ $2, \epsilon_{\text {elec }}=50 \mathrm{~nJ} / \mathrm{bit} /$ and $\epsilon_{a m p}=100 \mathrm{pJ} / \mathrm{bit} / \mathrm{m}^{2}$.

It is easy to observe that the energy balanced scheme outperforms the traditional hop-by-hop transmission scheme especially when $R$ is small. Compared with hop-by-hop transmission, the lifetime has been prolonged nearly $400 \%$ when $R=5 \mathrm{~m}$. Even when $R=10 \mathrm{~m}$, the network lifetime has been doubled. However, the improvement degrades with the increase of hop distance, and the reason for this behavior can be explained as follows. With the increase of hop distance $R$, the distance from the nodes to the sink becomes large. According to our energy model, the direct transmission will consume much energy and becomes energy inefficient. To balance energy consumption, only a small part of packets will be directly transmitted to the sink, which means that most packets are delivered via hop-by-hop transmission.

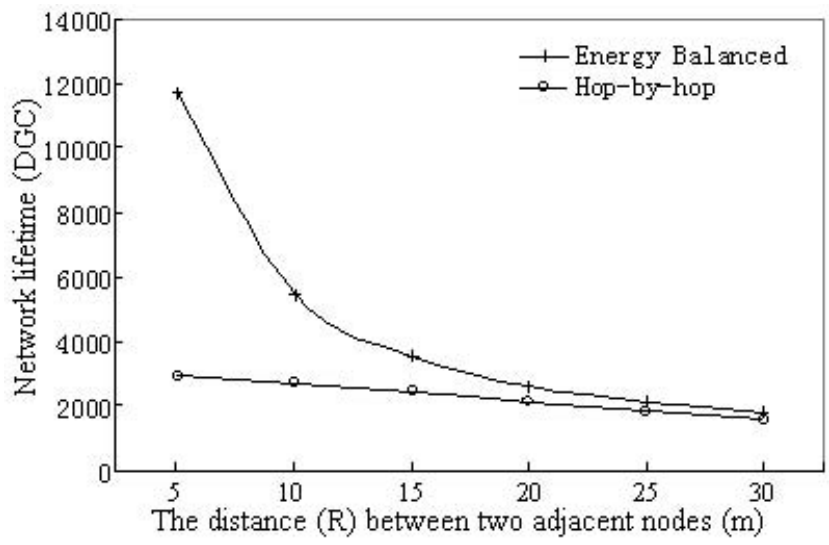

Figure 8. Lifetime comparison for a $R L N$ with 100 nodes.

\section{Application to Heterogenous Cluster-based Sensor Networks}

In our scheme, energy balancing is achieved by intelligently controlling the ratio between direct transmission and hop-by-hop transmission. However, such policy has a limited effect. When network becomes larger, for the nodes located far away from the sink, direct transmission is energy expensive and most packets will be delivered to the sink through hop-by-hop transmission (see Fig.3). Although our scheme still outperforms the hop-by-hop transmission scheme with respect to large-scale networks, the network lifetime extension becomes small, which can also be easily observed from Fig.8. Another practical consideration deserved to note is that most realistic sensor motes usually have limited transmission range. For the case where the sink is out of the maximal transmission range of some sensor nodes, our scheme may be not applicable. However, we argue that this drawback can be efficiently solved by integrating our scheme with clustering techniques.

Hierarchical (clustering) technique has been shown as an energy efficient scheme for large-scale networks. Instead of using the flat communication pattern, the sensors are organized into small clusters. Each cluster has a cluster head which is responsible for coordination among the nodes within its cluster and communication with other cluster heads or the sink. Although cluster heads rotation scheme has been proposed to balance energy consumption, our scheme has more advantages than cluster heads rotation scheme. First, the transmission probability allocated to each node only needs to be computed once, and the communication complexity for computing such optimal transmission probability is very low. More importantly, the processing and communication overheads incurred by cluster heads reelection have been completely avoided. 


\section{Conclusions}

In this article, we investigate the problem of balancing energy consumption to maximize network lifetime for data gathering sensor networks. The combination of hop-byhop transmission and direct transmission is exploited and we formulate the energy consumption balancing problem as an optimal transmission probability allocation problem. We first discuss this problem for chain networks and propose full distributed solutions which exchange only $O(1)$ messages per-channel. The solution to balance energy consumption in general-topology sensor networks is derived by dividing the network into sections and mapping it onto chain models. We also derive the optimal number of sections in terms of maximizing network lifetime. Numerical results reveal that our scheme outperforms the conventional hop-by-hop transmission scheme which has been shown more energy efficient than long distance transmission. Finally, we extend our solution to heterogeneous cluster-based sensor networks. We show that our scheme is more efficient than cluster heads rotation scheme because the overhead incurred by cluster heads re-election has been fully avoided. Moreover, by integrating with clustering techniques, the drawbacks of our scheme can be overcomed, and network lifetime can be prolonged to a significant extent.

\section{References}

[1] H. Bai, M. Atiquzzaman, and D. Lilja. Wireless sensor network for aircraft health monitoring. In Proceedings of the First International Conference on Broadband Networks (BROADNETS2004), pages 748-750, 2005.

[2] D. M. Blough and P. Santi. Investigating upper bounds on network lifetime extension for cell-based energy conservation techniques in stationary ad hoc networks. In Proceedings of the Eighth Annual International Conference on Mobile Computing and Networking (MOBICOM2002), pages 183-192, 2002.

[3] A. Cerpa, J. Elson, D. Estrin, L. Girod, M. Hamilton, and J. Zhao. Habitat monitoring: Application driver for wireless communications technology. In Proceedings of ACM SIGCOMM Workshop on Data Communications, pages 20-41, 2001.

[4] A. Cerpa and D. Estrin. ASCENT: Adaptive self-configuring sensor networks topologies. IEEE Transactions on Mobile Computing, 3:272-285, 2004.

[5] V. Dam, Tijs, and K. Langendoen. An adaptive energyefficient mac protocol for wireless sensor networks. In Proceedings of the First ACM Conference on Embedded Networked Sensor Systems, pages 171-180, 2003.

[6] C. Efthymiou, S. Nikoletseas, and J. Rolim. Energy balanced data propagation in wireless sensor networks. In Proceedings of the 18th International Parallel and Distributed Processing Symposium(IPDPS2004), page 225a, 2004.
[7] W. R. Heinzelman, A. Chandrakasan, and H. Balakrishnan. Energy-efficient communication protocol forwireless microsensor networks. In Proceedings of the 33rd Hawaii International Conference on System Sciences, pages 4-7, 2000.

[8] T. Hou, Y. Shi, and H. Sherali. Rate allocation in wireless sensor networks with network lifetime requirement. In Proceedings of ACM International Symposium on Mobile Ad Hoc Networking and Computing (MobiHoc2004), pages 6777, 2004.

[9] I. Howitt and J. Wang. Energy balanced chain in distributed networks. In Proceedings of IEEE Wireless Communications and Networking Conference 2004 (WCNC2004), pages 1721-1726, 2004

[10] M. Kohvakka, M. Hannikainen, and T. D. Hamalainen. Wireless sensor network implementation for industrial linear position metering. In Proceedings of the 8th Euromicro Conference on Digital System Design(DSD2005), pages 267-275, 2005.

[11] J. C. Koo. Traffic monitoring using sensor networks:vehicle detection strategies. Technical report, http://www.stanford.edu/ jckoo/ee359/, 2004.

[12] Q. Li, J. Aslam, and D. Rus. Online power-aware routing in wireless ad-hoc networks. In Proceedings of the 7th Annual International Conference on Mobile Computing and Networking (Mobicom2001), pages 97-107, 2001.

[13] T. Melodia, D. Pompili, and I. F. Akyildiz. Optimal local topology knowledge for energy efficient geographical routing in sensor networks. In Proceedings of the 24th Conference on Computer Communications (Infocom2004), 2004.

[14] S. Olariu and I. Stojmenovic. Design guidelines for maximizing lifetime and avoiding energy holes in sensor networks with uniform distribution and uniform reporting. In Proceedings of the 25th Conference on Computer Cотmunications (INFOCOM2006), 2006.

[15] M. Perillo, Z. Cheng, and W. Heinzelman. On the problem of unbalanced load distribution in wireless sensor networks. In Proceedings of the IEEE GLOBECOM Wireless Ad Hoc and Sensor Networks, 2004.

[16] Y. Wang, H. Wu, R. Nelavelli, and N.-F. Tzeng. Balancebased energy-efficient communication protocols for wireless sensor networks. In Proceedings of the International Workshop on Wireless Ad hoc and Sensor Networks (WWASN2006), 2006.

[17] Y. Xue and B. Li. A location-aided power-aware routing protocol in mobile ad hoc networks. In Proceedings of the 44th annual IEEE Global Telecommunications Conference, pages 2837-2841, 2001.

[18] T. Ye and S. Kalyanaraman. A recursive random search algorithm for large-scale network parameter configuration. In Proceedings of ACM SIGMETRICS international conference on Measurement and modeling of computer systems, pages 196-205, 2003.

[19] W. Ye, J. Heidemann, and D. Estrin. Medium access control with coordinated, adaptive sleeping for wireless sensor networks. ACM/IEEE Transactions on Networking, 12:493506, 2004. 